\title{
The characterization of turbulent heat and moisture transport during a gust-front event over the Indian peninsula
}

Subharthi Chowdhuri ( $\sim$ subharthi.cat@tropmet.res.in )

Indian Institute of Tropical Meteorology https://orcid.org/0000-0002-5518-7701

Kiran Todekar

Indian Institute of Tropical Meteorology

Thara V Prabha

Indian Institute of Tropical Meteorology

\section{Research Article}

Keywords: Atmospheric surface layer, Gust-front, Heat and moisture transport, Information theory,

Turbulence organization

Posted Date: April 12th, 2021

DOl: https://doi.org/10.21203/rs.3.rs-408500/v1

License: (c) (1) This work is licensed under a Creative Commons Attribution 4.0 International License.

Read Full License 


\title{
1 The characterization of turbulent heat and moisture transport during a gust-front event over the Indian peninsula
}

\author{
4 Subharthi Chowdhuri - Kiran Todekar . \\ 5 Thara V Prabha
}

7 Received: date / Accepted: date
Subharthi Chowdhuri · Kiran Todekar . Thara V Prabha Indian Institute of Tropical Meteorology

Dr. Homi Bhaba Road, Pashan, Pune-411008

Ministry of Earth Sciences, India

E-mail: subharthi.cat@tropmet.res.in, ORCID: 0000-0002-5518-7701 
$31 \quad$ Article highlights

32 - The topology of temperature and moisture structures show a sharp contrast between the active and quiescent regimes.

- The turbulent time scales of temperature and moisture vary in a power-law manner with different regime-wise exponents.

- The structural dissimilarity between the two scalars affects the heat and moisture transport between the two regimes.

\section{Introduction}

The boundary-layer exchange of heat and moisture has an astounding influence on the convection in the tropics and especially during the cold pool events 26. 32. Initiation of isolated convection is favored when the land surface is dry and depending on the moisture in the lower atmosphere and in the soil, wet conditions facilitate large organized convective clusters. The evaporative cooling of rain from such cloud clusters and the associated downdrafts initiate a cold pool, which introduces cold and moist air into the dry and sub-saturated boundary layer. As the moist air interfaces with the dry surrounding air, the density differences between the two air masses create the gust-front, characterized by strong upward motions and heterogeneity in temperature and moisture. The development of cold pools indeed contribute to changes in the spatio-temporal patterns of the surface fluxes. Most of our understanding on the cold pools and the related impacts on the surface fluxes are from the mid-latitude systems 19, 31. These studies have revealed that the cold pools can significantly alter the turbulence properties of the atmospheric surface layer (ASL) by causing a significant reduction in temperature, which often lead to the formation of stable boundary layers. However, the modulations of surface fluxes due to the cold pools are still to be explored over the Indian subcontinent, where detailed measurements of ASL turbulence are somewhat lacking.

To close this gap, a detailed micrometeorological observation system was established over the rain shadow region of the Western Ghat as part of the Cloud Aerosol Interaction and Precipitation Enhancement Experiment (CAIPEEX), in an effort to understand the linkage between the cloud processes and the surface-atmosphere exchanges. By using CAIPEEX datasets from a C-Band Doppler weather radar and 50-m micrometeorological tower, Chowdhuri et al. [12] studied the impact of a gust-front event on the ASL turbulence. They discovered that, similar to mid-latitude studies [27, 33, the incidence of the gust front was followed by a convergence of cold air pool, which in turn affected the turbulent temperature structure in the ASL. Due to such event, two distinct regimes were visible, where in one regime the turbulent temperature fluctuations remained quite intense analogous to daytime convective periods, whereas in the second regime the fluctuations diminished significantly as in stable conditions. Additionally, Chowdhuri et al. 12] demonstrated that the time scales of the temperature fluctuations displayed a power-law behavior 
whose exponents changed between the two regimes. By drawing an analogy with self-organized criticality in complex systems [2, 20, 1, they interpreted this as, the deep-convective cells whose outflows generated the gust front, acted as an external stimuli which disturbed its surroundings beyond the tipping point and created a scale-free response. Accordingly, this response propagated to the surface layer of the convective boundary layer and generated turbulent structures having self-similar size distributions.

The aforementioned results were illuminating to judge the structural characteristics of the turbulent temperature fluctuations in the ASL, as the gust-front traversed the tower location. However, in that particular study, they did not consider the effect of these structures on the turbulent heat and moisture transport. Undoubtedly, such investigation is of fundamental interest, given the importance of heat and moisture fluxes in sustenance of convection associated with the gust-front. Moreover, it is timely as well, since to the best of our knowledge no prior information is available on these aspects over the Indian region. Therefore, one may ask,

1. Whether there is any structural similarity in the temperature and moisture fluctuations between the active and quiescent regimes?

2. How the structures in the scalar fluctuations interact with the vertical velocity to generate signatures in their fluxes?

3. Can such structural interaction explain any regime-wise dissimilarity between the heat and moisture fluxes?

In this article, we attempt to answer these questions through novel data analysis techniques employed on the same 50-m micrometeorological dataset from CAIPEEX, used in Chowdhuri et al. [12. During our presentation, we arrange the paper in three different sections. In Sect. 2, we provide a brief description of the field-experimental dataset, in Sect. 3 we discuss the results, and lastly in Sect. 4 we summarize the key findings and lay out the scope for further research.

\section{Dataset description}

To investigate the characteristics of the turbulent heat and moisture fluxes during the gust-front event, we used the same micrometeorological dataset from a 50-m instrumented tower as described in Chowdhuri et al. [12]. This tower was erected over a non-irrigated and nearly-flat grassland in Solapur, India $\left(17.6^{\circ}\right.$ $\mathrm{N}, 75.9^{\circ} \mathrm{E}, 510 \mathrm{~m}$ above mean sea level), equipped with time-synchronized eddy-covariance (EC) systems at four levels with heights $z=4,8,20$, and $40 \mathrm{~m}$ ( $z$ is the height above the ground). Each EC system comprised of a sonic anemometer (Windmaster-Pro, Gill instruments, UK) and an open-path $\mathrm{CO}_{2}-\mathrm{H}_{2} \mathrm{O}$ gas analyzer (LI-7542, Li-cor Inc., USA). The horizontal separation between the sonic anemometer and gas analyzer was approximately $20 \mathrm{~cm}$ towards the East and $7 \mathrm{~cm}$ towards the North. Due to the close proximity, there was hardly any time-lag between the sonic anemometer and gas analyzer measurements. 
The data from these four EC systems were synchronized in time through GPS clocks and sampled continuously at 10-Hz frequency, divided into 30-min intervals. Before using the water vapor data from the gas-analyzer, density corrections were carried out following the procedure listed in Detto and Katul [13. Moreover, no double-coordinate rotation was applied to the sonic-anemometer data which forces the mean vertical velocity to be zero [17. The reason for this is, the 30-min averaged non-zero vertical velocities may have arisen due to the presence of large-scale variability over the tower location [12. Further details about the tower instrumentation and site description can be found in Chowdhuri et al. [12].

\section{Results and discussion}

We begin with delineating the statistical properties of the turbulence structures which impact the heat and moisture fluxes during the passage of a gust-front. An occurrence of such event creates two distinct regimes, reminiscent of convective and nocturnal periods. To unveil the structural differences between these two regimes, the time scales of the turbulent motions are explored through persistence analysis. Subsequently, to assess the role of turbulence organization on the flux transport processes, a novel polar-quadrant based approach coupled with information theory is introduced. Furthermore, throughout the presentation, plausible physical interpretations are provided to explain the results.

\subsection{Structural description of turbulence in the two regimes}

\subsubsection{General features}

With an aim to characterize the vertical transport of heat and moisture, it is imperative to scrutinize the properties of the component signals (temperature/moisture and vertical velocity) which constitute these fluxes. In Fig. 1. we show the $10-\mathrm{Hz}$ time series of the sonic temperature $\left(T_{S}\right)$, water-vapor density $\left(\rho_{\mathrm{H}_{2} \mathrm{O}}\right)$, and vertical velocity $(w)$ as measured by the EC systems at $z=4,8$, 20, and $40 \mathrm{~m}$, between 14:00-16:10 PM (local solar time, GMT $+05: 30$ ). Note that, this period relates to the time when the gust-front passed over the tower location 12 .

Chowdhuri et al. 12 demonstrated that due to the cold-air outflow from the precipitating convective cells, a near-surface stable layer was established (early nightfall) which caused two clearly distinct regimes in the high-frequency sonic temperature measurements at all the four heights $(z=4,8,20$, and $40 \mathrm{~m})$. In the first regime (14:10-15:00 PM) large temperature changes were observed analogous to daytime convective turbulence. Whereas, in the second regime (15:10-16:10 PM), the intrusion of cold-air into the surface layer suppressed the variations in temperature, resulting in a quiescent signal typical of nocturnal 
turbulence. The gray-shaded regions in Fig. 1 represent these two regimes. From Figs. 1 a-d, one can notice that in the first regime the water-vapor densities (red lines, values shown at the right-hand-side of the $y$ axis) broadly show similar temporal patterns of ramp-cliff structures, concurrent with $T_{S}$ (blue lines, values shown at the left-hand-side of the $y$ axis). However, for the second regime, even though there is a drop in the $\rho_{\mathrm{H}_{2} \mathrm{O}}$ values, that occurs at a later time as opposed to $T_{S}$. On the other hand, for all the four levels no discernible change is observed in vertical velocity (blue lines in Figs. 1 1 - $h$ ) between these two regimes.

To further investigate the role of such different regime-wise behavior towards the transport of heat and moisture, it is important to segregate the turbulent fluctuations from the three signals. Previously, we computed the turbulent fluctuations in $T_{S}$ by removing a portion of the signal through applying a Fourier filter with a threshold frequency set at $0.01 \mathrm{~Hz}[12$. In the present study, we extended this procedure to all the three signals $\left(T_{S}, \rho_{\mathrm{H}_{2} \mathrm{O}}\right.$, and $w)$ to extract the turbulent fluctuations. The thick and dashed black lines in Figs. 1 $1 \mathrm{a}-\mathrm{d}$ show the respective low-frequency components of $T_{S}$ and $\rho_{\mathrm{H}_{2} \mathrm{O}}$, overlaid on the original ones. Likewise, for $w$, the solid black lines in Figs. 1p-h convey the same information. It is interesting to observe that, the low-frequency variations in $w$ get larger as the height increases. This is consistent with the expectation that the vertical velocities of the large-scale structures are blocked by the ground [24, 8]. Hereafter, the turbulent fluctuations in temperature, water-vapor, and vertical velocities are denoted as $T^{\prime}, \rho^{\prime}$, and $w^{\prime}$, respectively.

\subsubsection{Cross-correlation analysis}

By separating the turbulent part from the low-frequency trend, we explore how strongly the fluctuations in the scalars and vertical velocity at different measurement heights are related to one another. To accomplish that, we employ cross-correlation analysis and estimate the relative strength of the fluctuations at higher heights with respect to the lowest measurement level. This information is crucial to quantify the vertical coherence among the turbulent structures and see whether there is any change in such property between the two regimes. In Fig. 2, the cross-correlation coefficients between the two signals among different heights (either for $T^{\prime}, \rho^{\prime}$, or $w^{\prime}$ ) are presented individually for the two regimes. These coefficients have previously been used in several ASL studies to probe the vertical structure of turbulence [5, 22, 23] and can be evaluated mathematically as,

$$
R_{x y}^{s}(\tau)=\frac{\overline{s_{y}(t+\tau, z) s_{x}\left(t, z_{\mathrm{ref}}\right)}}{\sigma\left(s_{y}\right) \sigma\left(s_{x}\right)},
$$

where $s_{x}$ is the reference signal at $z_{\text {ref }}=4 \mathrm{~m}, s_{y}$ is the signal at higher heights $(z=8,20,40 \mathrm{~m})$ shifted through time, and $\tau$ is the time-lag. The $\tau$ values are either positive or negative, depending on whether $s_{y}$ leads or lags $s_{x}$. Note that, Eq. 1 is designed when $s_{y}$ lags $s_{x}$, but can also be used for a leading case by keeping $s_{y}$ fixed and moving $s_{x}$ ahead in time. 


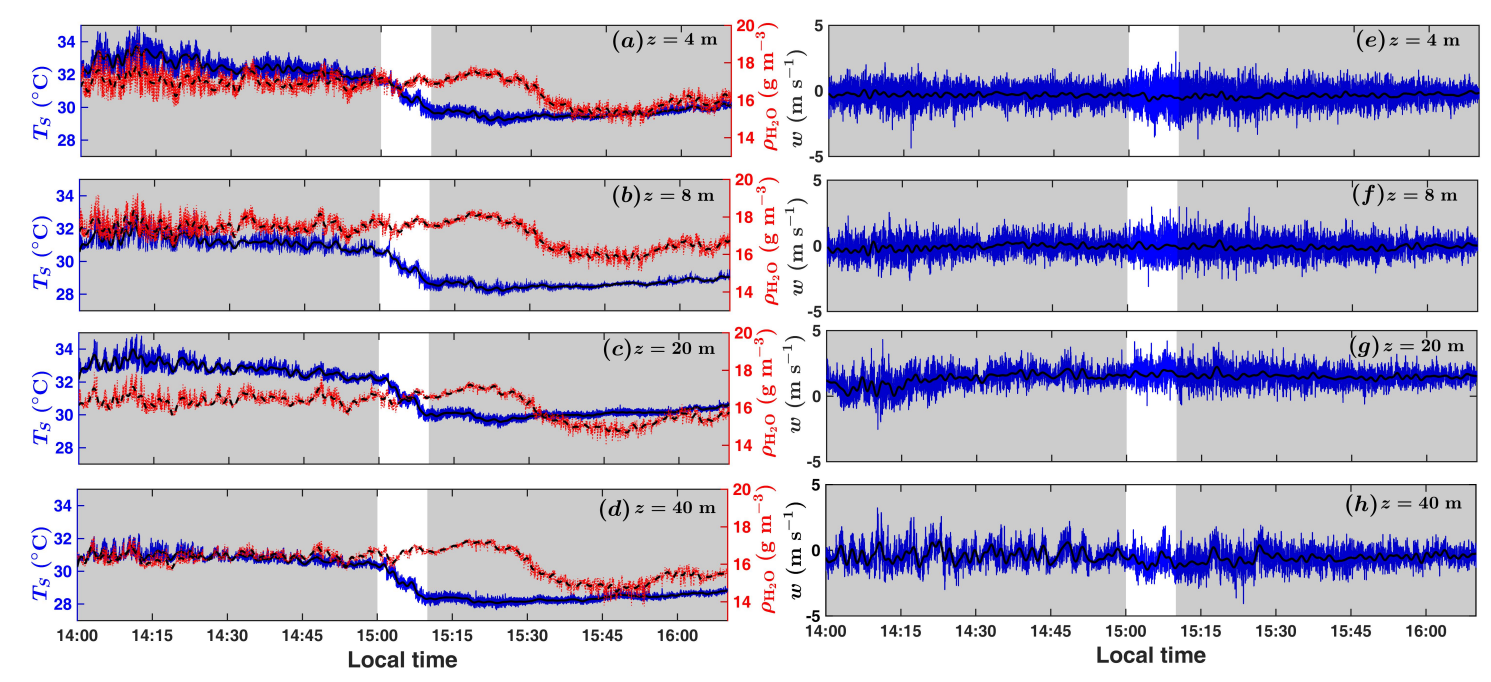

Fig. 1: The two hours time series of the sonic temperature $\left(T_{S}\right.$, blue lines) and water-vapor density $\left(\rho_{\mathrm{H}_{2} \mathrm{O}}\right.$, red lines) at a $10-\mathrm{Hz}$ sampling rate are shown in panels (a)-(d) from the four sonic anemometers $(z=4,8,20$, and $40 \mathrm{~m})$ corresponding to the period 14:00-16:10 PM. The $T_{S}$ and $\rho_{\mathrm{H}_{2} \mathrm{O}}$ values are represented on the left and right $y$ axes of (a)-(d), respectively. The solid and dashed black lines in (a)-(d) indicate a Fourier filtered low-frequency signal (threshold frequency set at $0.01 \mathrm{~Hz}$ ), related to $T_{S}$ and $\rho_{\mathrm{H}_{2} \mathrm{O}}$. In $(\mathrm{e})-(\mathrm{h})$, the $10-\mathrm{Hz}$ time series of the vertical velocity ( $w$, blue lines) are displayed for $z=4$, 8,20 , and $40 \mathrm{~m}$. The solid black lines in (e)-(h) depict the same low-frequency trend for $w$. The gray-shaded regions in all the panels designate the two periods between 14:00-15:00 PM and 15:10-16:10 PM, which occurred before and after the passage of the gust-front.

For the first regime, the cross-correlation coefficients of $w^{\prime}\left(R_{x y}^{w^{\prime}}(\tau)\right)$ decrease as the measurement heights increase (Fig. 2a). Apart from that, the peaks in $R_{x y}^{w^{\prime}}(\tau)$ are clustered around the zero-lag, signifying a negligible shift with height. But, for $T^{\prime}$ and $\rho^{\prime}$, a prominent shift towards the positive $\tau$ values is observed in their cross-correlation peak positions as $z$ gets larger (Figs. 2b-c). However, in the second regime, $R_{x y}^{w^{\prime}}(\tau)$ and $R_{x y}^{\rho^{\prime}}(\tau)$ behave almost identically as in the first regime (Figs. $2 \mathrm{~d}$ and $\mathrm{f}$ ). Conversely, for $T^{\prime}, R_{x y}^{T^{\prime}}(\tau)$ values decrease with no apparent shift in their peak positions, as the second regime is encountered (Fig. 2p).

To put these above results into perspective, it is prudent to recognize that the shifts in the peak positions of the cross-correlation coefficients are associated with inclination angles of the turbulent structures, apparently caused due to the presence of vertical wind shear [5]. For this particular gust-front case, Chowdhuri et al. [12] have shown that a substantial amount of wind shear was 

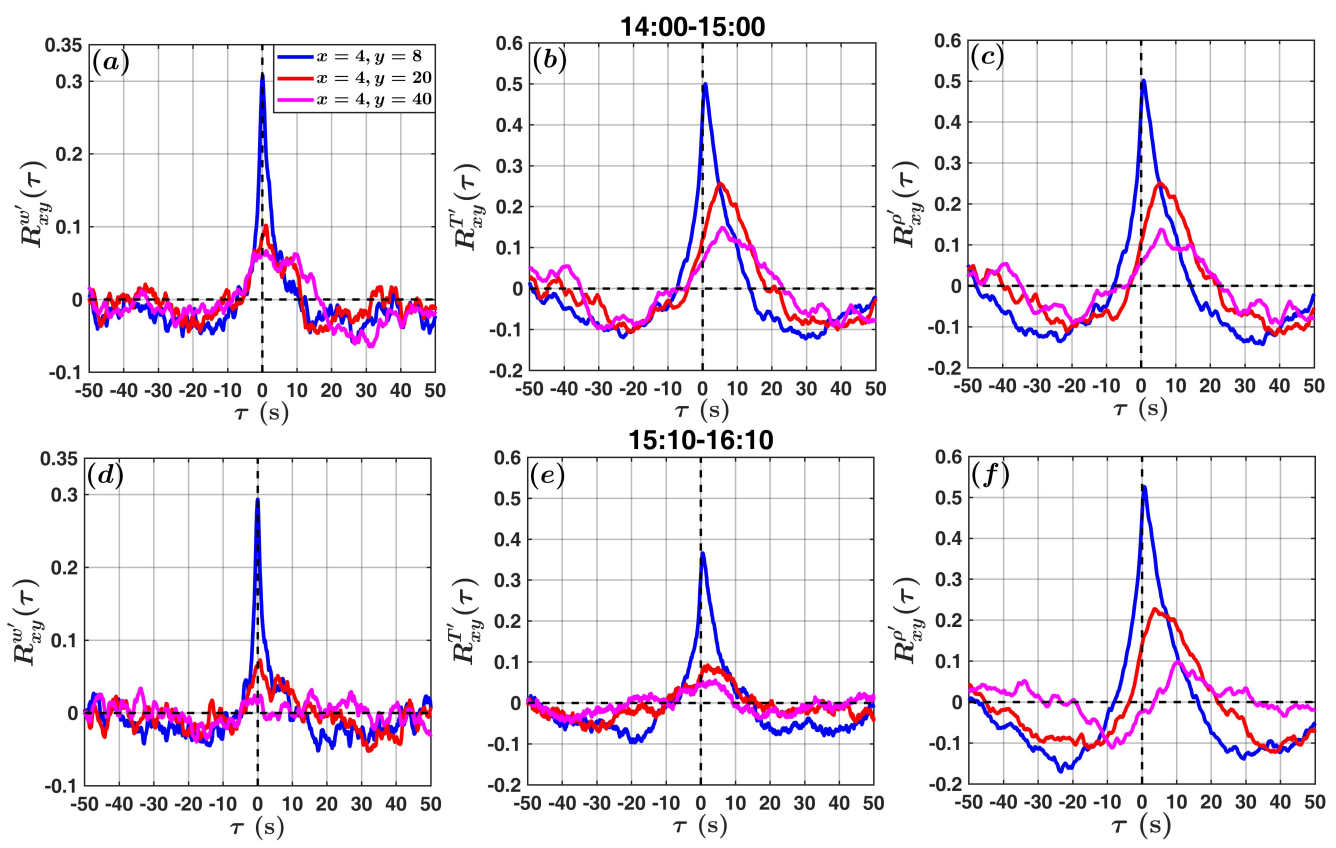

Fig. 2: The cross-correlation coefficients $\left(R_{x y}\right)$ between the 4-m $(x=4 \mathrm{~m})$ and other three observation levels $(y=8,20$, and $40 \mathrm{~m})$ are plotted against the time-lags $(\tau)$ for the (a) vertical velocity $\left(w^{\prime}\right),(\mathrm{b})$ sonic temperature $\left(T^{\prime}\right)$, and (c) water-vapor density $\left(\rho^{\prime}\right)$ fluctuations. The description of different colored lines is provided in the legend of (a). The top panels represent information for the period between 14:00-15:00 PM, whereas the bottom panels show the same but for 15:10-16:10 PM.

present during the period between 14:00-16:10 PM (see their Fig. 4). Thus, the observed zero-shifts in $R_{x y}^{w^{\prime}}(\tau)$ (Figs. $2 \mathrm{p}$ and d) indicate that the turbulent structures which govern $w^{\prime}$ are immune to the effect of wind-shear and remain statistically invariant with the changes in the regimes. Contrarily, the effects of wind-shear on the vertical orientation of the turbulent structures related to $T^{\prime}$ and $\rho^{\prime}$ are different between the two regimes. For the first regime, a significant inclination exists in both the structures which affect the turbulent fluctuations in temperature and water-vapor (Figs. 2 $2 \mathrm{~b}-\mathrm{c}$ ). Whereas, in the second regime, the inclination almost disappears for temperature, while it is intact for the water-vapor (Figs. 2 2 -f). This is a remarkable result with serious implications towards the heat and moisture transport, as will be revealed in the subsequent sections. 

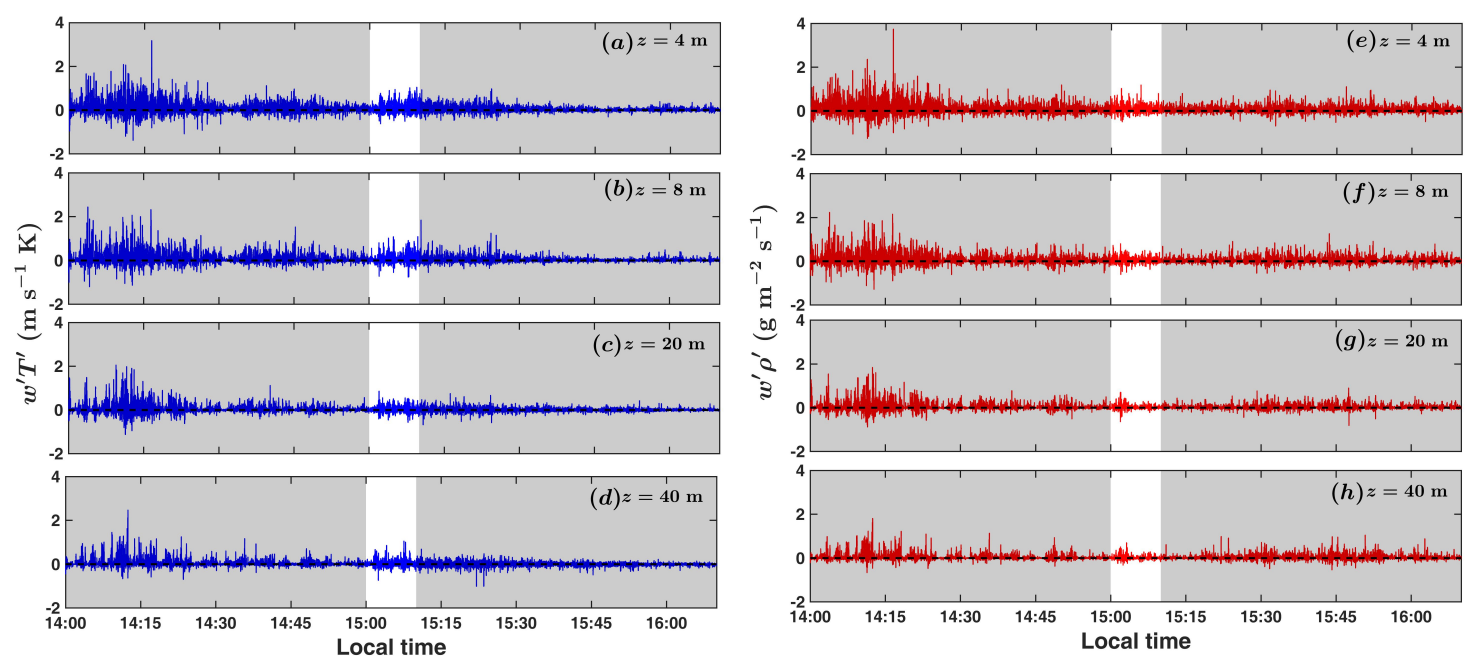

Fig. 3: The turbulent fluxes of heat $\left(w^{\prime} T^{\prime}\right)$ are shown in panels (a)-(d) from the four sonic anemometers $(z=4,8,20$, and $40 \mathrm{~m})$ corresponding to the period 14:00-16:10 PM. In (e)-(h) the information is presented for the water-vapor fluxes $\left(w^{\prime} \rho^{\prime}\right)$. The gray-shaded regions carry the same meaning as in Fig. 1

\subsubsection{Heat and moisture fluxes}

From the aforementioned discussion, we infer that the fluctuations in temperature and water-vapor are generated due to similar turbulent structures in the first regime. However, as the second regime is approached, a structural disparity prevails between the two scalars, although such difference happens to be nearly absent in $w^{\prime}$. Therefore, a complex interaction between turbulent structures with contrasting vertical orientations influence the characteristics of the heat and water-vapor fluxes $\left(w^{\prime} T^{\prime}\right.$ and $\left.w^{\prime} \rho^{\prime}\right)$ at all the four measurement heights. To gain more insight into what type of flux signatures are generated due to such interaction, in Fig. 3 the instantaneous time-series of $w^{\prime} T^{\prime}$ and $w^{\prime} \rho^{\prime}$ are shown at $z=4,8,20$, and $40 \mathrm{~m}$.

During the first regime, across all the four heights, one can see occasional occurrences of large bursts (intense activities lasting for a small time) in both the heat and water-vapor fluxes (Fig. 3). These kinds of bursts in the scalar fluxes, found for the first regime, are regarded as a prevalent feature of convective turbulence 14, 4, 18, 28, 11. Nevertheless, in the second regime, the bursts become exceedingly rare for $w^{\prime} T^{\prime}$ (Fig. 33-d), while still being present in $w^{\prime} \rho^{\prime}$ with reduced intensities (Fig. 33-h). To examine further about the scales of turbulent motions which cause such intermittent behavior in the heat and moisture fluxes, we present results from persistence analysis [6, 7, 11] in Sect. 3.2 
3.2 Identification of the scales of turbulent motions

\subsubsection{Persistence analysis}

Persistence is a concept widely applied in non-equilibrium statistical mechanics and defined as the probability that the local value of a fluctuating field does not change its sign for a certain amount of time [3, 15. In other words, the persistence timescale $t_{p}$ is the time up to which a signal stays positive or negative, before switching its sign (see Chowdhuri et al. 6, 7] for a brief review). For wall-bounded turbulence, several studies have shown that the distributions of the persistence time scales can be interpreted as equivalent to the streamwise size distributions (by applying Taylor's hypothesis) of the turbulent structures in such flows [30, 16, 29, 25. In convective ASL flows, Chowdhuri et al. [6, 11] have illustrated that the persistence analysis is an effective tool to provide a structural description behind the intermittent fluctuations in velocity and temperature, and in the associated heat and momentum fluxes. Additional details about the computation of persistence probability density functions (PDFs) are laid out in Chowdhuri et al. [6].

Chowdhuri et al. [12] showed through persistence analysis that the passage of the gust-front created a scale-free response, which generated self-similar structures affecting the turbulent temperature fluctuations at all the four measurement heights [12]. To comprehend the role of these structures towards the transport of heat and moisture, we present the persistence PDFs of $T^{\prime}, \rho^{\prime}$, $w^{\prime}, w^{\prime} T^{\prime}$, and $w^{\prime} \rho^{\prime}$ in Fig. 4 . The persistence time scales are denoted by $t_{p}$ and to document any discrepancies, the top and bottom panels in Fig. 4 display the persistence PDFs $\left(P\left(t_{p}\right)\right.$ separately for each of the two regimes. Note that, a log-log representation is used in these plots, so any power-law emerges as a straight line.

In Figs. 4 a $-\mathrm{e}$, one could spot roughly a similar power-law behavior $\left(P\left(t_{p}\right) \propto\right.$ $\left.t_{p}{ }^{-2.1}\right)$ in all the five variables, with hardly any difference among different heights. Physically, it indicates that in the first regime the statistical characteristics of both the scalars, vertical velocities, and the associated fluxes are all governed by the same self-similar structures at all the heights, although their vertical orientations depend differently on the wind-shear (Fig. $2 a-c$ ). On the other hand, in the second regime, a significant disparity is observed in $P\left(t_{p}\right)$ between the temperature and moisture. In Figs. 4 - $\mathrm{g}$, the exponent of the power-law in $P_{T^{\prime}}\left(t_{p}\right)$ is equal to -2.5 (shown in thick green lines), whereas for $P_{\rho^{\prime}}\left(t_{p}\right)$ the exponent remains close to the first regime $(-2.1$, shown in dashdotted gray lines). This outcome reinforces our statement about Figs. 2p-f, i.e., the topology of the structures which govern the variations in moisture do not alter their attributes despite the observation that the temperature structures exhibit a behavioral change between the two regimes. Interestingly, for $w^{\prime}, w^{\prime} T^{\prime}$, and $w^{\prime} \rho^{\prime}$ the exponents in $P\left(t_{p}\right)$ are comparable to $T^{\prime}$ (Figs. $4 \mathrm{~h}-\mathrm{j}$ ), suggesting that the transport of heat and moisture are primarily accomplished through those turbulent structures which have identical effects on the temperature and vertical velocity fluctuations. 

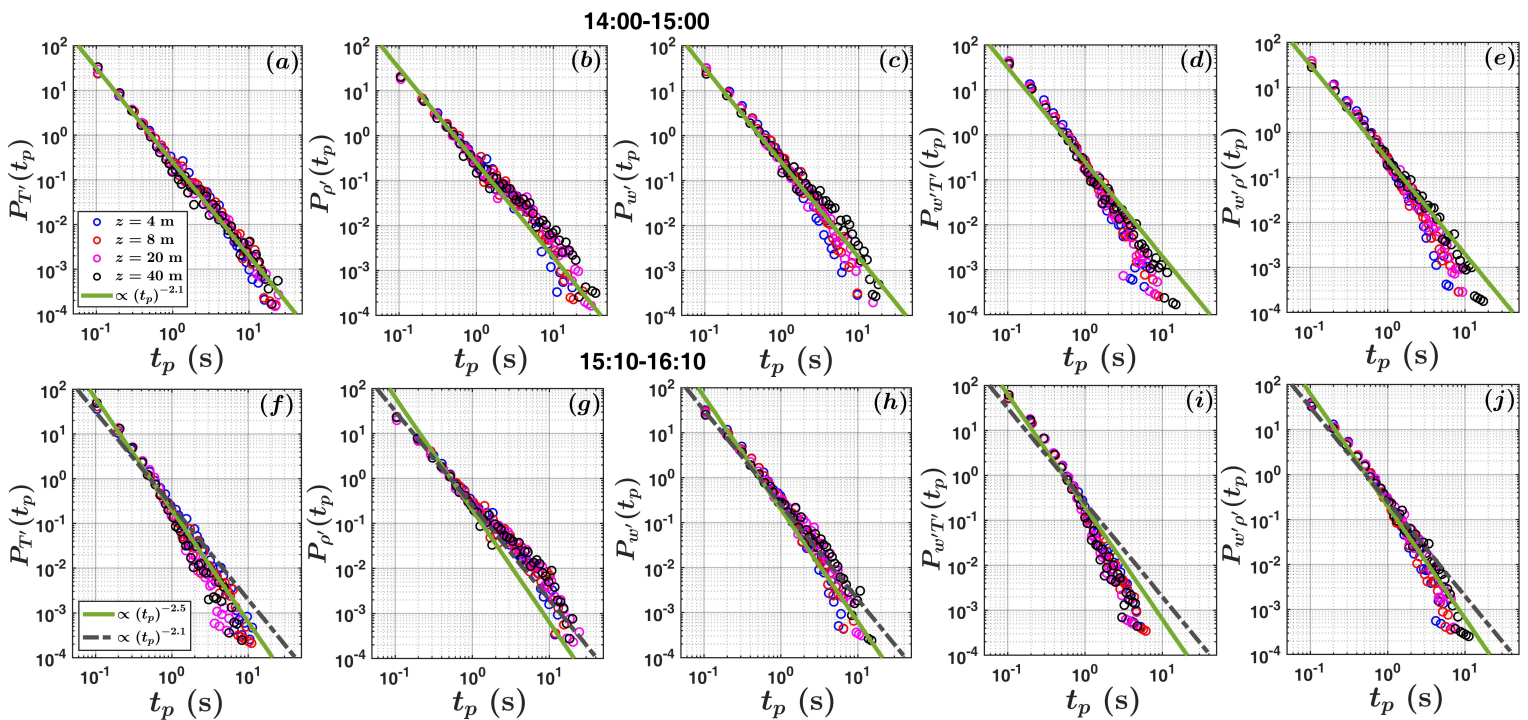

Fig. 4: The persistence probability density functions (PDFs, $P\left(t_{p}\right)$ ) of (a) $T^{\prime}$, (b) $\rho^{\prime}$, (c) $w^{\prime}$, (d) $w^{\prime} T^{\prime}$, and (e) $w^{\prime} \rho^{\prime}$ are plotted against the time scales $t_{p}$. A description of different markers depicting the four different heights is provided in the legend of (a). The solid green lines in (a)-(e) denote a best-fit power-law with exponent -2.1 , associated with the persistence PDFs of $T^{\prime}$. The top panels represent information about the first regime (14:00-15:00 PM), whereas the bottom panels represent the second regime (15:10-16:10 PM). The solid green lines in (f)-(j) display an another best-fit power-law of $T^{\prime}$ with exponent -2.5 , computed for the second regime. For comparison purpose, the dash-dotted gray lines in (f)-(j) show the same power-law of $T^{\prime}$ with exponent -2.1 , corresponding to the first regime (14:00-15:00 PM).

\subsubsection{Amplitude PDFs}

Notwithstanding the fact that persistence analysis is a convenient method to describe the sizes of the intermittent patterns which affect the turbulent signals, it remains insensitive to the fluctuation amplitudes [11]. It is thus instructive to see the amplitude signatures associated with the structures whose geometrical features have been explored till now (Figs. 2 and 4 ). To disseminate such information, in Fig. 5 we show the PDFs of temperature, moisture, vertical velocity, and the scalar fluxes for the two regimes. In order to better highlight the height variations in the statistical properties of the turbulent fluctuations, no normalization has been performed while computing the PDFs. It is immediately noticeable that the $w^{\prime}$ PDFs collapse for all the four heights, with nearly no change in the shapes between the two regimes (Figs. 5. and h). For $T^{\prime}$ and $\rho^{\prime}$ PDFs, a little variation with height can be detected in the first regime (Figs. $5 \mathrm{a}-\mathrm{b}$ ). However, in the second regime, the 

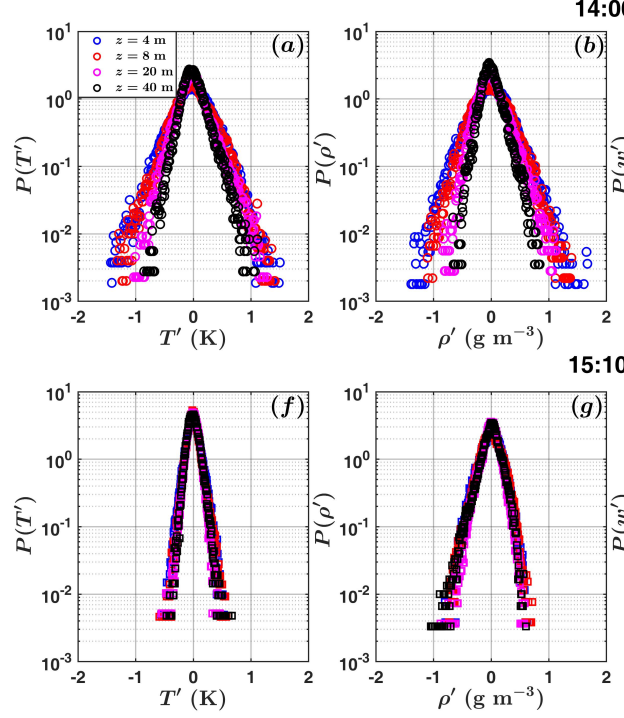

15:10-16:10
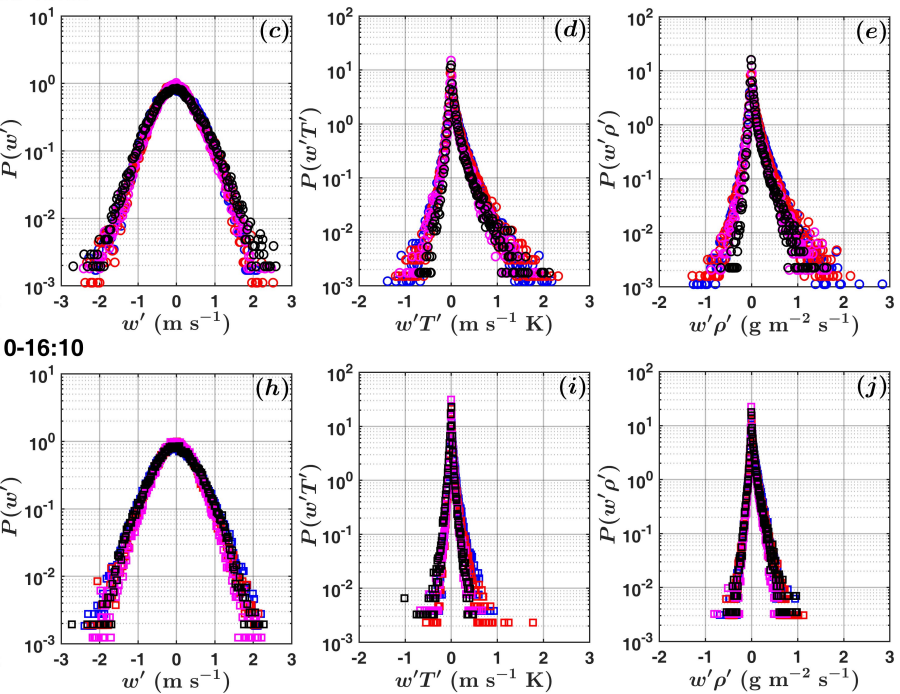

Fig. 5: The PDFs of (a) $T^{\prime}$, (b) $\rho^{\prime}$, (c) $w^{\prime}$, (d) $w^{\prime} T^{\prime}$, and (e) $w^{\prime} \rho^{\prime}$ are shown. A description of different markers depicting the four different heights is provided in the legend of (a). The bottom panels (f-j) represent the same information as in the top panels (a-e) but for the period 15:10-16:10 PM.

height dependence disappears for temperature and moisture. In addition to that, only a narrow range of amplitudes dominate the PDFs of temperature, while the range being slightly larger for moisture (Figs. 5f-g). A similar impact of the two regimes could also be observed on the scalar flux PDFs. The heat and moisture fluxes remain substantially skewed to the positive values in Figs. $5 \mathrm{~d}-\mathrm{e}$ (first regime), but become largely attenuated in the second regime as depicted in Figs. 5 - j.

By combining results from the persistence analysis and amplitude PDFs, we infer that in the first regime heat and moisture are both transported by topologically similar turbulent structures which mostly give rise to the positive values in the fluxes. Conversely, for the other regime, the turbulent fluctuations associated with temperature and moisture remain structurally quite different, although their signatures on the flux amplitudes appear to be similar. To elucidate more on the connection between the organized turbulent motions and scalar flux transport, one may ask:

1. What type of turbulent motions cause burst-like activities in the fluxes corresponding to the first regime?

2. What is the role of turbulence organization towards the heat and moisture transport efficiencies associated with the two regimes? 
To answer those, in Sect. 3.3 we introduce a polar-quadrant based approach through which the characteristics of the scalar fluxes are evaluated in terms of organized structures in the flow.

3.3 The impact of turbulence organization on the heat and moisture transport

\subsubsection{Polar-quadrant analysis}

Generally, quadrant analysis is regarded as a standard technique to quantify the contributions of organized motions in the turbulent fluxes [34. In this approach, usually the flux fractions and time fractions from each quadrant are reported to assess the relative importance of the various turbulent motions, associated with different flow structures [21, 10, 9]. The normal practice while performing quadrant analysis is to choose a Cartesian co-ordinate system where the $x$ and $y$ axes denote the fluctuations in the two variables. However, in this representation, a fundamental question remain unanswered, i.e., what governs the strength of the coupling between the two turbulent signals owing to which the fluxes exist?
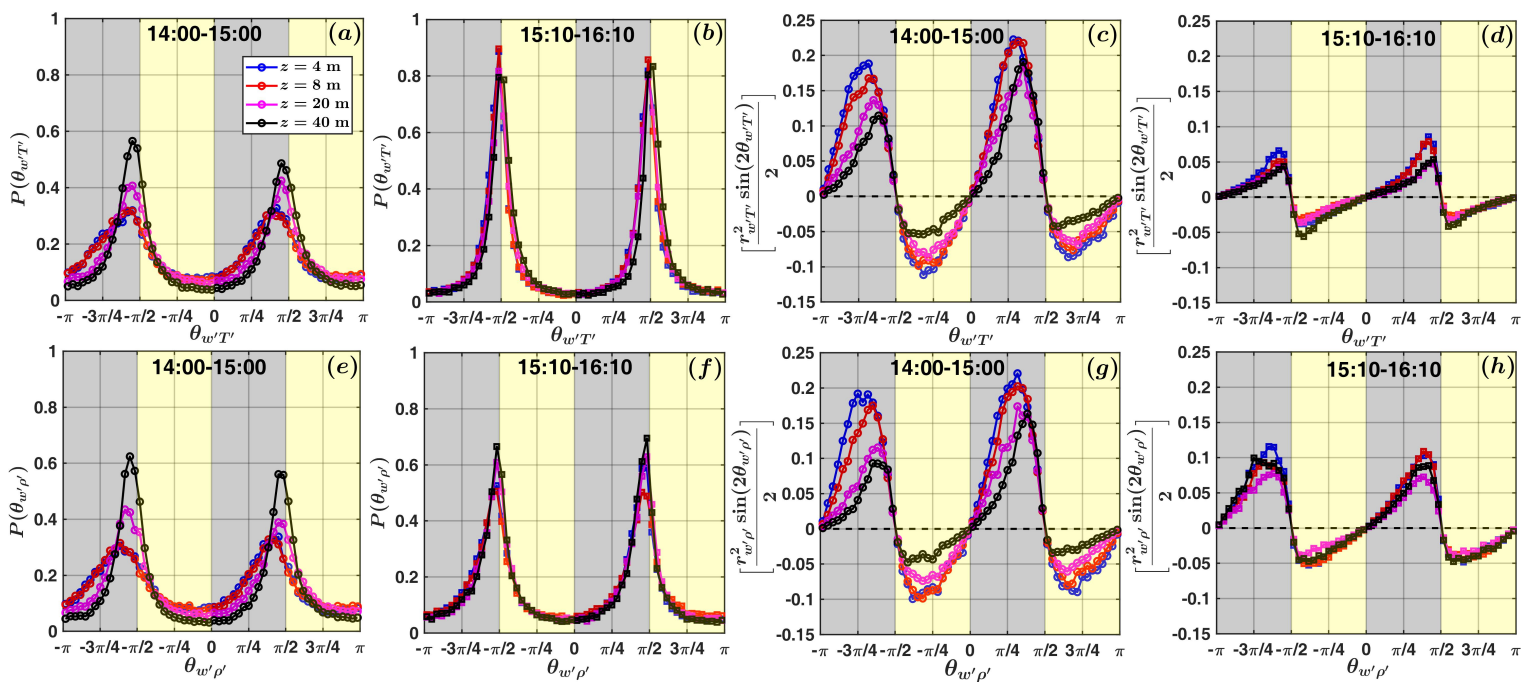

Fig. 6: The phase angle PDFs of $T^{\prime}-w^{\prime}$ and $\rho^{\prime}-w^{\prime}$ quadrants $\left(P\left(\theta_{w^{\prime} T^{\prime}}\right)\right.$ and $\left.P\left(\theta_{w^{\prime} \rho^{\prime}}\right)\right)$ are shown in panels (a), (b), (e), and (f), corresponding to the two periods 14:00-15:00 PM and 15:10-16:10 PM. The amount of heat and moisture fluxes $\left(w^{\prime} T^{\prime}\right.$ and $\left.w^{\prime} \rho^{\prime}\right)$ associated with such phase angles (see Eq. 44 are presented in panels (c), (d), (g), and (h). The gray- and yellow-shaded regions in all the panels show the locations in the phase angle space which constitute the down-gradient and counter-gradient quadrants. 
To resolve this issue, Chowdhuri et al. [11] proposed a novel method where they designated each point in the quadrant plane with two parameters, the phase angles and amplitudes (for a graphical demonstration see their Fig. $4)$. They used a polar co-ordinate system to define the phase angles $(\theta)$ and amplitudes $(r)$ as,

$$
\begin{array}{r}
\theta_{w^{\prime} x^{\prime}}=\arctan \left(w^{\prime} / x^{\prime}\right), \\
r_{w^{\prime} x^{\prime}}=\sqrt{{w^{\prime}}^{2}+x^{\prime 2}},
\end{array}
$$

where $x^{\prime}$ is the other turbulent variable (for this study $T^{\prime}$ or $\rho^{\prime}$ ) apart from $w^{\prime}$ which constitutes the flux. The phase angles vary between $-\pi$ to $\pi$ and their ranges are related to the four different quadrants (see Table 1 from Chowdhuri et al. [11]). In the polar co-ordinate system, the instantaneous fluxes associated with each point are expressed as,

$$
w^{\prime} x^{\prime}=\frac{1}{2} r_{w^{\prime} x^{\prime}}^{2} \sin \left(2 \theta_{w^{\prime} x^{\prime}}\right),
$$

with the detailed derivation being provided in Chowdhuri et al. 11. Besides that, the PDFs of the phase angles $P\left(\theta_{w^{\prime} x^{\prime}}\right)$ are related to the time-fractions $\left(T_{f}\right)_{\mathrm{X}}$ spent in each quadrant $\mathrm{X}$ as,

$$
\left(T_{f}\right)_{\mathrm{X}}=\int_{-\pi}^{\pi} P\left(\theta_{w^{\prime} x^{\prime}}\right) I_{\mathrm{X}}\left(\theta_{w^{\prime} x^{\prime}}\right) d \theta_{w^{\prime} x^{\prime}},
$$

where $I_{\mathrm{X}}\left(\theta_{\hat{w} \hat{x}}\right)$ is an identity function which is unity when $\theta_{w^{\prime} x^{\prime}}$ lies within quadrant $\mathrm{X}$ or zero elsewhere. Based on these formulations, it is possible to ascertain the properties of the turbulent motions occurring in each quadrant by investigating the phase angle PDFs and the corresponding flux amplitudes. In Fig. 6 such information is displayed for the present case in hand. The grayand yellow-shaded regions in Fig. 6 illustrate the motions occurring in the down-gradient and counter-gradient quadrants, respectively. The flux values plotted in Figs. 6r, d, g, and h are computed from Eq. 4 and averaged between the same phase angle bins used to estimate $P\left(\theta_{w^{\prime} T^{\prime}}\right)$ and $P\left(\theta_{w^{\prime} \rho^{\prime}}\right)$ (see Figs. $6 \mathrm{a}, \mathrm{b}, \mathrm{e}$, and $\mathrm{f})$.

By inspecting the phase angle PDFs, one can see that in the first regime $P\left(\theta_{w^{\prime} T^{\prime}}\right)$ and $P\left(\theta_{w^{\prime} \rho^{\prime}}\right)$ behave in a nearly similar fashion (Figs. 6a and e). Two peaks are observed in the PDFs, corresponding to the ejection $\left(0 \leq \theta_{w^{\prime} x^{\prime}} \leq \pi / 2\right)$ and sweep $\left(-\pi \leq \theta_{w^{\prime} x^{\prime}} \leq-\pi / 2\right)$ motions, whose heights increase with $z$. Moreover, the peak heights of $P\left(\theta_{w^{\prime} T^{\prime}}\right)$ related to the sweeps are somewhat larger in number than the ones related to the ejections. At the same time, from the flux amplitude plots, we notice that the fluxes associated with the ejection motions exceed the ones associated with the sweeps (Figs. 6c and g). Therefore, the ejections occur a little less frequently than sweeps but are accompanied with large intensities, causing burst-like activities in the fluxes as observed in the first regime (Fig. 33. It is intriguing to note that, in both the phase angle PDFs and flux amplitudes, the variations with $z$ appear to be more prominent for the sweep motions rather than for the ejections. 
Even though the physics behind such phenomenon is elusive at present, the phase angle PDFs and flux amplitudes become almost independent of $z$ in the second regime. The phase angle PDFs of $\theta_{w^{\prime} T^{\prime}}$ attain two peaks at $\pm \pi / 2$, suggesting near-zero transport of heat in that regime (Fig. 6b). This is confirmed with Fig. 6d, where the heat flux amplitudes are significantly diminished as compared to Fig. 6. (first regime). On the other hand, the peak positions of $P\left(\theta_{w^{\prime} \rho^{\prime}}\right)$ remain slightly shifted from $\pm \pi / 2$ (Fig. 6f), thus yielding higher moisture flux values than heat (Fig. 6h).
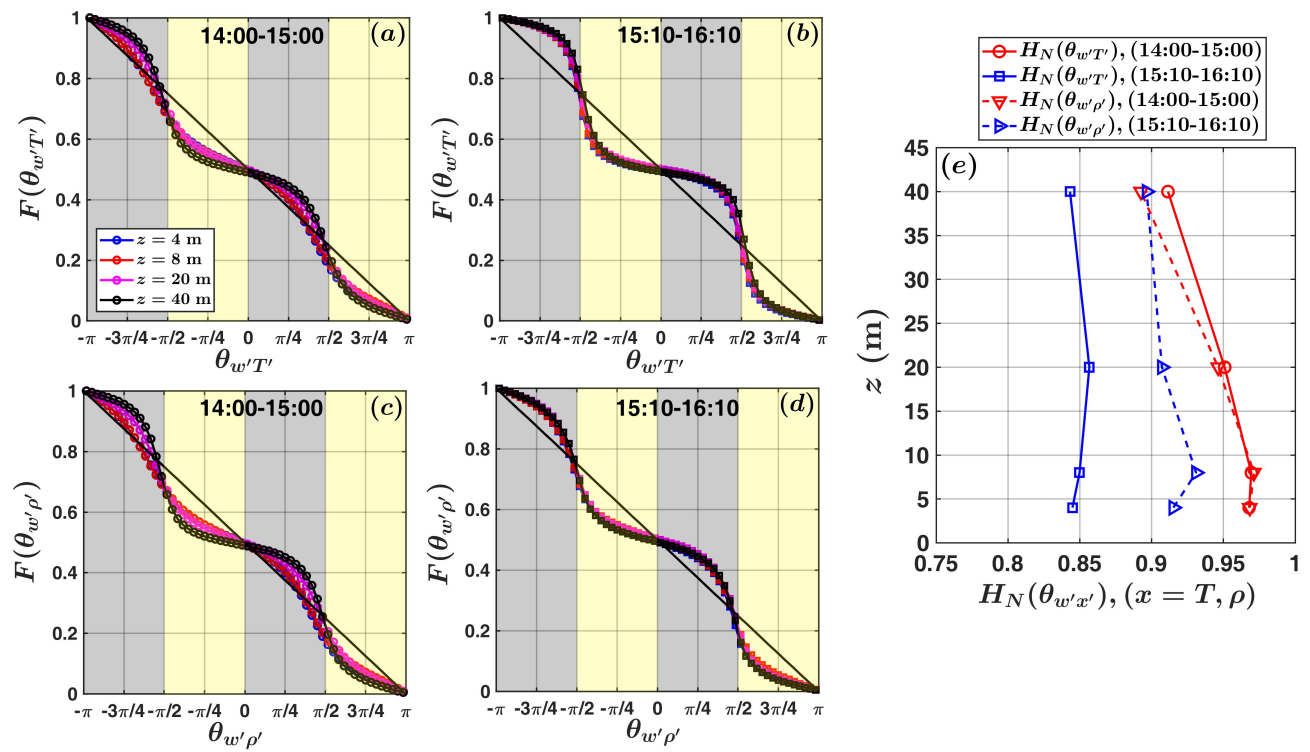

Fig. 7: The CDFs of $T^{\prime}-w^{\prime}$ and $\rho^{\prime}-w^{\prime}$ phase angles $\left(F\left(\theta_{w^{\prime} T^{\prime}}\right)\right.$ and $\left.F\left(\theta_{w^{\prime} \rho^{\prime}}\right)\right)$ are shown in panels (a)-(d), corresponding to the two regimes (14:00-15:00 PM and 15:10-16:10 PM). The shaded regions convey the same information as in Fig. 6. The solid black lines indicate the CDFs of a uniform distribution. In (e), the vertical profiles of the normalized Shannon entropy of the phase angles $\left(H_{N}\left(\theta_{w^{\prime} T^{\prime}}\right)\right.$ and $\left.H_{N}\left(\theta_{w^{\prime} \rho^{\prime}}\right)\right)$ are displayed for the two regimes. The thick and dashed red (blue) lines denote the $H_{N}$ values from the first (second) regime, as described in the legend of (e).

\subsubsection{Information entropy}

Overall, the results in Fig. 6 provide a detailed description about the relative roles of ejection and sweep motions in the scalar flux transport, commensurate with the gust-front event. Nevertheless, it remains unclear whether the discrepancies observed in the heat and moisture fluxes between the two regimes are in 
any way linked to different organizational structure of turbulence. In order to evaluate that, one can compare the information entropy of the phase angles for both heat and moisture, corresponding to the two regimes. Chowdhuri et al. 11] theoretically showed that if in Eq. 4 the amplitudes $r$ are considered to be independent of $\theta$ and the PDFs $P(\theta)$ resemble a uniform distribution (i.e., the phase angles are randomly oriented with no order whatsoever), then the time-averaged flux becomes zero under such constraints. As a consequence, the departure of the phase angle PDFs from a uniform distribution could be used to quantify the role of turbulence organization on the flux transport, given the assumption that the information about $r$ remains largely irrelevant [11].

Therefore, it is appropriate to investigate whether the phase angle PDFs of heat and moisture fluxes deviate from a uniform distribution in the two regimes. For this purpose, in Figs. $7 \mathrm{a}-\mathrm{d}$ we show the cumulative distribution functions (CDFs) of $\theta_{w^{\prime} T^{\prime}}$ and $\theta_{w^{\prime} \rho^{\prime}}\left(F\left(\theta_{w^{\prime} x^{\prime}}\right)\right)$, since in such plots the uniform distribution appears as a straight line (solid black lines). In the first regime, both $F\left(\theta_{w^{\prime} T^{\prime}}\right)$ and $F\left(\theta_{w^{\prime} \rho^{\prime}}\right)$ closely follow the uniform distribution at all the four heights (Figs. $7 \mathrm{a}$ and c). Conversely, the CDFs of the same phase angles deviate more from the solid black lines in the second regime (Figs. $7 \mathrm{~b}$ and d). Following Chowdhuri et al. [11, to measure the deviation we compute the Shannon entropy of the phase angles as,

$$
H_{N}\left(\theta_{w^{\prime} x^{\prime}}\right)=-\frac{1}{\ln \left(N_{\mathrm{b}}\right)} \sum_{i=1}^{N_{\mathrm{b}}} P_{i}\left(\theta_{w^{\prime} x^{\prime}}\right) \ln \left[P_{i}\left(\theta_{w^{\prime} x^{\prime}}\right)\right],
$$

where $N_{\mathrm{b}}$ is the number of bins in which the $\theta_{w^{\prime} x^{\prime}}$ values are divided (60 in our case), and $P_{i}\left(\theta_{w^{\prime} x^{\prime}}\right)$ is the probability of occurrence of a particular binned value $\theta_{w^{\prime} x^{\prime}}$. For a uniform distribution, $H_{N}\left(\theta_{w^{\prime} x^{\prime}}\right)$ in Eq. 6 is equal to 1, given $P_{i}\left(\theta_{w^{\prime} x^{\prime}}\right)=1 / N_{\mathrm{b}}$ for all the bin indexes. Hence, the departure from unity in $H_{N}\left(\theta_{w^{\prime} x^{\prime}}\right)$ illustrates that the orientation of the phase angles differs from a random configuration. In Fig. 77, the vertical profiles of $H_{N}\left(\theta_{w^{\prime} x^{\prime}}\right)$ are presented for the same two periods. One can observe that, $H_{N}\left(\theta_{w^{\prime} T^{\prime}}\right)$ and $H_{N}\left(\theta_{w^{\prime} \rho^{\prime}}\right)$ are close to unity for all the $z$ values (red lines in Fig. $7 \mathrm{e}$ ), consistent with Figs. $7 \mathrm{a}$ and c. Contrarily, for the other regime, $H_{N}\left(\theta_{w^{\prime} \rho^{\prime}}\right)$ values do not change much from 1 , while $H_{N}\left(\theta_{w^{\prime} T^{\prime}}\right)$ shows a pronounced deviation (blue lines in Fig. 7e).

At a first glance, the Shannon entropies of the phase angles dictate that in the first regime the flux transporting motions from the four quadrants exhibit quasi-random patterns. According to the model of Chowdhuri et al. [1], this configuration transports very little flux when the amplitudes play no role. However, from Fig. 6 it is evident that in the first regime a substantial amount of heat and moisture is carried by the ejection and sweep motions. To explain this conundrum, the amplitude information must be invoked. By doing so, it becomes clear that in the first regime a strong coupling exists between the amplitudes and phases of $T^{\prime}\left(\rho^{\prime}\right)$ and $w^{\prime}$ which strengthens the heat (moisture) transport. On the other hand, in the second regime, the role of such coupling is different for heat and moisture. As an instance, in this 
regime, $H_{N}\left(\theta_{w^{\prime} T^{\prime}}\right)$ values display a strong deviation from unity accompanied with almost no transport of heat. A far-from-random organization of the phase angles would have increased the flux, but the phases and amplitudes of $T^{\prime}$ and $w^{\prime}$ remain coupled in a way so that small heat flux values are observed in the second regime (Figs. 3 and 6). But, for moisture, the flux values are larger than $w^{\prime} T^{\prime}$, while $H_{N}\left(\theta_{w^{\prime} \rho^{\prime}}\right)$ is close to unity. This indicates, the nature of coupling between the phases and amplitudes of $\rho^{\prime}$ and $w^{\prime}$ is nearly-similar to the first regime, causing enhancement in the moisture fluxes.

By condensing all the details rendered so far, we deduce that the regime-wise distinction between the heat and moisture originates due to two main reasons. First, the topology of the turbulent structures which affect $T^{\prime}$ and $\rho^{\prime}$ disagrees between the two regimes. Second, the phase and amplitude coupling of the flux-transporting motions associated with the presence of such structures, also display opposing behavior as the regime transition occurs. We present our conclusions in the next section.

\section{Conclusion}

For the first time over an Indian peninsula, we address the impact of a gustfront passage on the turbulent heat and moisture fluxes by using multi-level high-frequency measurements from a 50-m micrometeorological tower. A coldpool event associated with the gust-front separates two turbulence regimes, where in one the temperature fluctuations are intense (first regime) and in the other those remain subdued (second regime). In order to evaluate the structural features of turbulent motions which determine the heat and moisture transport characteristics in these two regimes, we employ advanced statistical techniques, such as cross-correlation, persistence, and polar-quadrant analyses. The results obtained from these methods are directed towards providing answers to the research questions posed in the introduction and can be summarized as:

1. The vertical orientations of the turbulent structures corresponding to the temperature and moisture fluctuations differ significantly between the two regimes. In the first regime, the structures remain vertically inclined in a similar fashion for both temperature and moisture. However, in the second regime, such inclination disappears for temperature while being retained for moisture.

2. In addition to the vertical inclination, the horizontal sizes of the turbulent structures (obtainable from persistence time scales using Taylor's hypothesis) show a conspicuous regime-wise distinction between the temperature and moisture. The sizes of temperature structures in both the regimes follow a power-law albeit with different exponents. On the other hand, the moisture structures too display a power-law behavior, but their exponents remain regime-invariant and equal to the temperature structures from the first regime.

3. The observed difference in such topology has a profound influence on the organized motions (ejections and sweeps) which govern the dissimilarity 
between the scalar fluxes in the two regimes. By employing a polar-quadrant based approach, it is discovered that in the first regime, the efficient transport of heat and moisture is tied to the fact that the phases and amplitudes of the flux-transporting motions remain strongly coupled. Nevertheless, in the second regime, the phase and amplitude coupling between the temperature and vertical velocity gets altered in such a way that it induces a substantial decrease in the heat flux. Conversely, for moisture and vertical velocity, the nature of coupling is nearly identical to the first regime, causing an enhancement in the fluxes.

To conclude, the physical insights gained from this study are useful in developing new parametrizations of turbulent heat and moisture fluxes, which would aid better simulations of the gust-front dynamics. The issue of momentum transport is not addressed in this work, given an inherent difficulty of defining the streamwmise and cross-stream axes under the presence of non-stationary flow features as the gust-front traverses the tower location. In our future endeavors, we wish to push the envelope by tackling the problem of momentum transport, leading towards further advancements in surface drag formulations related to the gust-front.

\section{Data availability}

On reasonable request, the EC dataset analyzed during the current study can be made available to the interested researchers by contacting Thara V Prabha (thara@tropmet.res.in). The computer codes needed to reproduce the figures are available by contacting Subharthi Chowdhuri at subharthi.cat@tropmet.res.in

\section{Conflict of Interest}

The authors declare that they have no conflict of interest.

\section{Author contributions}

Subharthi Chowdhuri and Thara V Prabha conceptualized the study. The data collection was performed by Subharthi Chowdhuri and Kiran Todekar. All the analyses were carried out by Subharthi Chowdhuri. The first draft was written by Subharthi Chowdhuri and the others commented on previous versions of the manuscript. All authors read and approved the final manuscript.

\section{Acknowledgements}

CAIPEEX is conducted by the Indian Institute of Tropical Meteorology, which is an autonomous institute and fully funded by the Ministry of Earth Sciences, 
Government of India. Authors are grateful to several colleagues who contributed to the success of the CAIPEEX project. The authors extend their heartfelt gratitude to Dr. Anand K Karipot who helped immensely with the datacollection, instrument set-ups, quality checks, and critical scientific suggestions. The authors also acknowledge the local support and hospitality provided by N. B. Navale Sinhgad College of Engineering (NBNSCOE), Kegaon-Solapur, during the experiment. The author Subharthi Chowdhuri thanks Dr. Tirtha Banerjee for many insightful discussions on persistence and polar-quadrant analyses.

\section{References}

1. Açıkalın, S. N. and Artun, E. C. (2017). The concept of self-organized criticality: The case study of the Arab uprising. In International Symposium on Chaos, Complexity and Leadership, pages 73-85. Springer.

2. Bak, P., Tang, C., and Wiesenfeld, K. (1988). Self-organized criticality. Phys. Rev. A, 38(1):364. https://doi.org/10.1103/PhysRevA.38.364

3. Bray, A., Majumdar, S., and Schehr, G. (2013). Persistence and firstpassage properties in nonequilibrium systems. Adv. Phys., 62(3):225-361. https://doi.org/10.1080/00018732.2013.803819.

4. Caramori, P., Schuepp, P., Desjardins, R., and MacPherson, I. (1994). Structural analysis of airborne flux estimates over a region. J. Clim., 7(5):627640. https://doi.org/10.1175/1520-0442(1994)007<0627:SAOAFE $>2.0$ $\mathrm{CO} ; 2$.

5. Chauhan, K., Hutchins, N., Monty, J., and Marusic, I. (2013). Structure inclination angles in the convective atmospheric surface layer. Boundary-Layer Meteorol., 147(1):41-50. https://doi.org/10.1007/s10546-012-9777-7.

6. Chowdhuri, S., Kalmár-Nagy, T., and Banerjee, T. (2020a). Persistence analysis of velocity and temperature fluctuations in convective surface layer turbulence. Phys. Fluids, 32(7):076601. https://doi.org/10.1063/5.0013911.

7. Chowdhuri, S., Kumar, S., and Banerjee, T. (2020b). Revisiting the role of intermittent heat transport towards Reynolds stress anisotropy in convective turbulence. J. Fluid Mech., 899:A26. https://doi.org/10.1017/jfm.2020 471.

8. Chowdhuri, S., McNaughton, K. G., and Prabha, T. V. (2019). An empirical scaling analysis of heat and momentum cospectra above the surface friction layer in a convective boundary layer. Boundary-Layer Meteorol., 170(2):257284. https://doi.org/10.1007/s10546-018-0397-8.

9. Chowdhuri, S. and Prabha, T. (2019). An evaluation of the dissimilarity in heat and momentum transport through quadrant analysis for an unstable atmospheric surface layer flow. Environ. Fluid Mech., 19(2):513-542. https: //doi.org/10.1007/s10652-018-9636-2

10. Chowdhuri, S., Prabha, T. V., Karipot, A., Dharamraj, T., and Patil, M. (2015). Relationship between the momentum and scalar fluxes close to the 
ground during the indian post-monsoon period. Boundary-Layer Meteorol., 154(2):333-348. https://doi.org/10.1007/s10546-014-9977-4.

11. Chowdhuri, S., Prabhakaran, T., and Banerjee, T. (2020c). Persistence behavior of heat and momentum fluxes in convective surface layer turbulence. Phys. Fluids, 32(11):115107. https://doi.org/10.1063/5.0027168.

12. Chowdhuri, S., Todekar, K., Murugavel, P., Karipot, A., and Prabha, T. V. (2021). Unravelling the turbulent structures of temperature variations during a gust front event: a case study. Environ. Fluid Mech., 21(1):263-281. https://doi.org/10.1007/s10652-020-09769-z.

13. Detto, M. and Katul, G. (2007). Simplified expressions for adjusting higher-order turbulent statistics obtained from open path gas analyzers. Boundary-Layer Meteorol., 122(1):205-216. https://doi.org/10.1007/ s10546-006-9105-1.

14. Duncan, M. and Schuepp, P. (1992). A method to delineate extreme structures within airborne flux traces over the FIFE site. J. Geophys. Res. Atmos., 97(D17):18487-18498. https://doi.org/10.1029/91JD03113

15. Ghannam, K., Nakai, T., Paschalis, A., Oishi, C., Kotani, A., Igarashi, Y., Kumagai, T., and Katul, G. (2016). Persistence and memory timescales in root-zone soil moisture dynamics. Water Resour. Res., 52(2):1427-1445. https://doi.org/10.1002/2015WR017983.

16. Kailasnath, P. and Sreenivasan, K. (1993). Zero crossings of velocity fluctuations in turbulent boundary layers. Phys. Fluids, 5(11):2879-2885. https://doi.org/10.1063/1.858697.

17. Kaimal, J. C. and Finnigan, J. J. (1994). Atmospheric boundary layer flows: Their structure and measurement. Oxford university press.

18. Katul, G., Albertson, J., Parlange, M., Chu, C., and Stricker, H. (1994). Conditional sampling, bursting, and the intermittent structure of sensible heat flux. J. Geophys. Res. Atmos., 99(D11):22869-22876. https://doi org/10.1029/94JD01679.

19. Lareau, N. P., Crosman, E., Whiteman, C. D., Horel, J. D., Hoch, S. W., Brown, W. O., and Horst, T. W. (2013). The persistent cold-air pool study. Bull. Amer. Meteor., 94(1):51-63. https://doi.org/10.1175/ BAMS-D-11-00255.1.

20. Lewis, T. G. (2010). Cause-and-effect or fooled by randomness? Homeland Security Affairs, 6(1). https://www.hsaj.org/articles/93

21. Li, D. and Bou-Zeid, E. (2011). Coherent structures and the dissimilarity of turbulent transport of momentum and scalars in the unstable atmospheric surface layer. Boundary-Layer Meteorol., 140(2):243-262. https://doi.org/ 10.1007/s10546-011-9613-5.

22. Liu, H.-Y., Bo, T.-L., and Liang, Y.-R. (2017). The variation of large-scale structure inclination angles in high Reynolds number atmospheric surface layers. Phys. Fluids, 29(3):035104. https://doi.org/10.1063/1.4978803

23. Lotfy, E. R. and Harun, Z. (2018). Effect of atmospheric boundary layer stability on the inclination angle of turbulence coherent structures. Environ. Fluid Mech., 18(3):637-659. https://doi.org/10.1007/ s10652-017-9558-4 
24. McNaughton, K., Clement, R., and Moncrieff, J. (2007). Scaling properties of velocity and temperature spectra above the surface friction layer in a convective atmospheric boundary layer. Nonlin. Processes Geophys., 14(3):257271. https://doi.org/10.5194/npg-14-257-2007.

25. Mora, D. O. and Obligado, M. (2020). Estimating the integral length scale on turbulent flows from the zero crossings of the longitudinal velocity fluctuation. Exp. Fluids, 61:199. https://doi.org/10.1007/ s00348-020-03033-2.

26. Pielke, R. A. (2001). Influence of the spatial distribution of vegetation and soils on the prediction of cumulus convective rainfall. Rev. Geophys., 39(2):151-177. https ://doi.org/10.1029/1999RG000072.

27. Rotunno, R., Klemp, J. B., and Weisman, M. L. (1988). A theory for strong, long-lived squall lines. J. Atmos. Sci., 45(3):463-485. https://doi org/10.1175/1520-0469(1988)045<0463: ATFSLL>2 .0.C0;2

28. Schmutz, M. and Vogt, R. (2019). Flux similarity and turbulent transport of momentum, heat and carbon dioxide in the urban boundary layer. Boundary-Layer Meteorol., 172(1):45-65. https://doi.org/10.1007/ s10546-019-00431-w.

29. Sreenivasan, K. and Bershadskii, A. (2006). Clustering properties in turbulent signals. J. Stat. Phys., 125(5-6):1141-1153. https://doi.org/10. 1007/s10955-006-9112-0.

30. Sreenivasan, K., Prabhu, A., and Narasimha, R. (1983). Zero-crossings in turbulent signals. J. Fluid Mech., 137:251-272. https://doi.org/10.1017/ S0022112083002396.

31. Sun, X. and Holmes, H. A. (2019). Surface turbulent fluxes during persistent cold-air pool events in the Salt Lake Valley, Utah. Part I: Observations. J. Appl. Meteorol. Clim., 58(12):2553-2568. https://doi.org/10.1175/ JAMC-D-19-0053.1

32. Tokinaga, H., Tanimoto, Y., Nonaka, M., Taguchi, B., Fukamachi, T., Xie, S.-P., Nakamura, H., Watanabe, T., and Yasuda, I. (2006). Atmospheric sounding over the winter Kuroshio extension: Effect of surface stability on atmospheric boundary layer structure. Geophys. Res. Lett., 33(4). https: //doi.org/10.1029/2005GL025102.

33. Torri, G., Kuang, Z., and Tian, Y. (2015). Mechanisms for convection triggering by cold pools. Geophys. Res. Lett., 42(6):1943-1950. https: //doi.org/10.1002/2015GL063227.

34. Wallace, J. (2016). Quadrant analysis in turbulence research: History and evolution. Annu. Rev. Fluid Mech., 48:131-158. https://doi.org/10.1146/ annurev-fluid-122414-034550. 

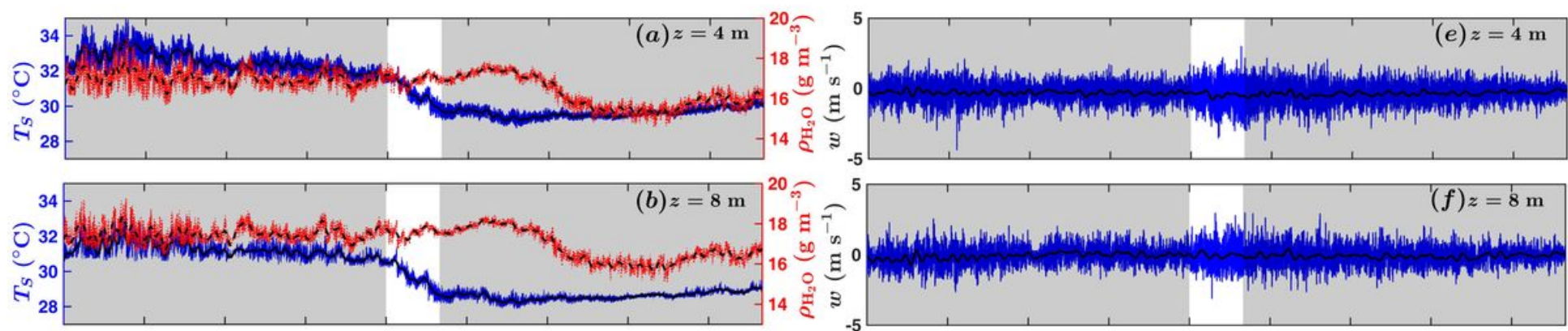

$(f) z=8 \mathrm{~m}$
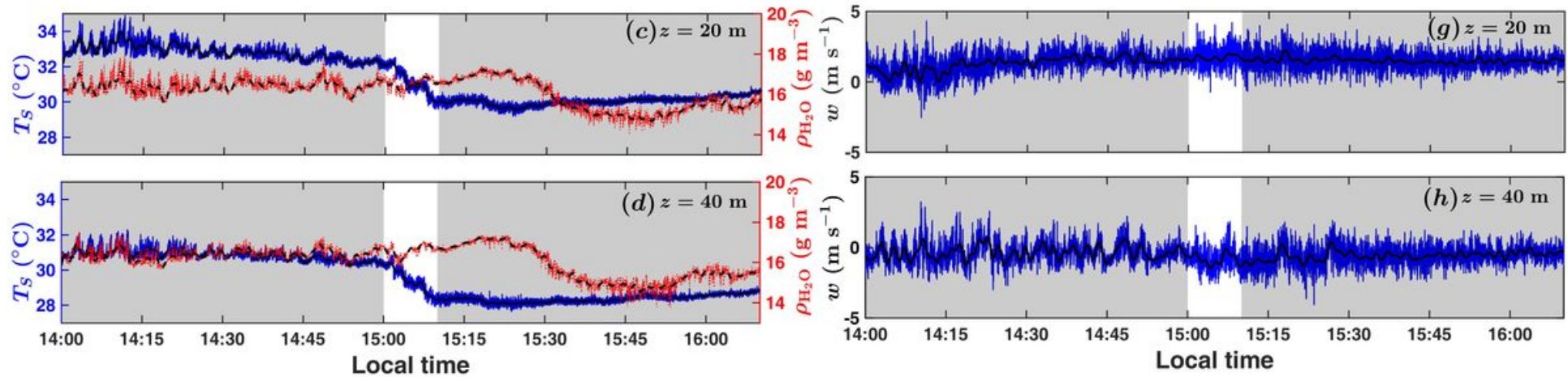

(h) $z=40 \mathrm{~m}$

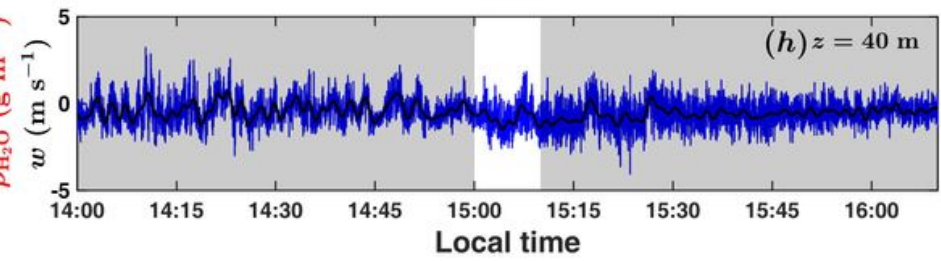

\section{Figure 1}

(see Manuscript file for full figure caption)
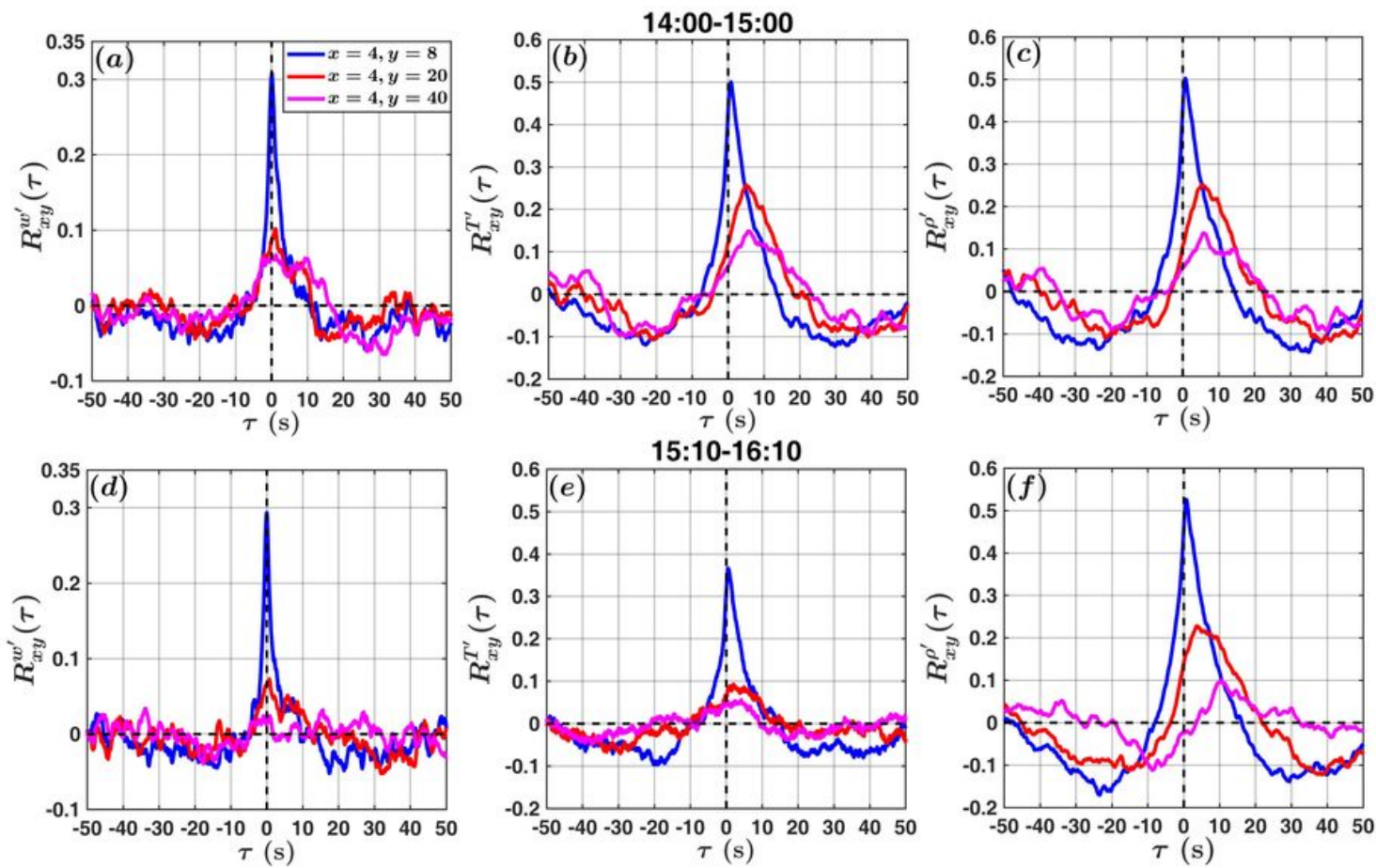

Figure 2

(see Manuscript file for full figure caption) 

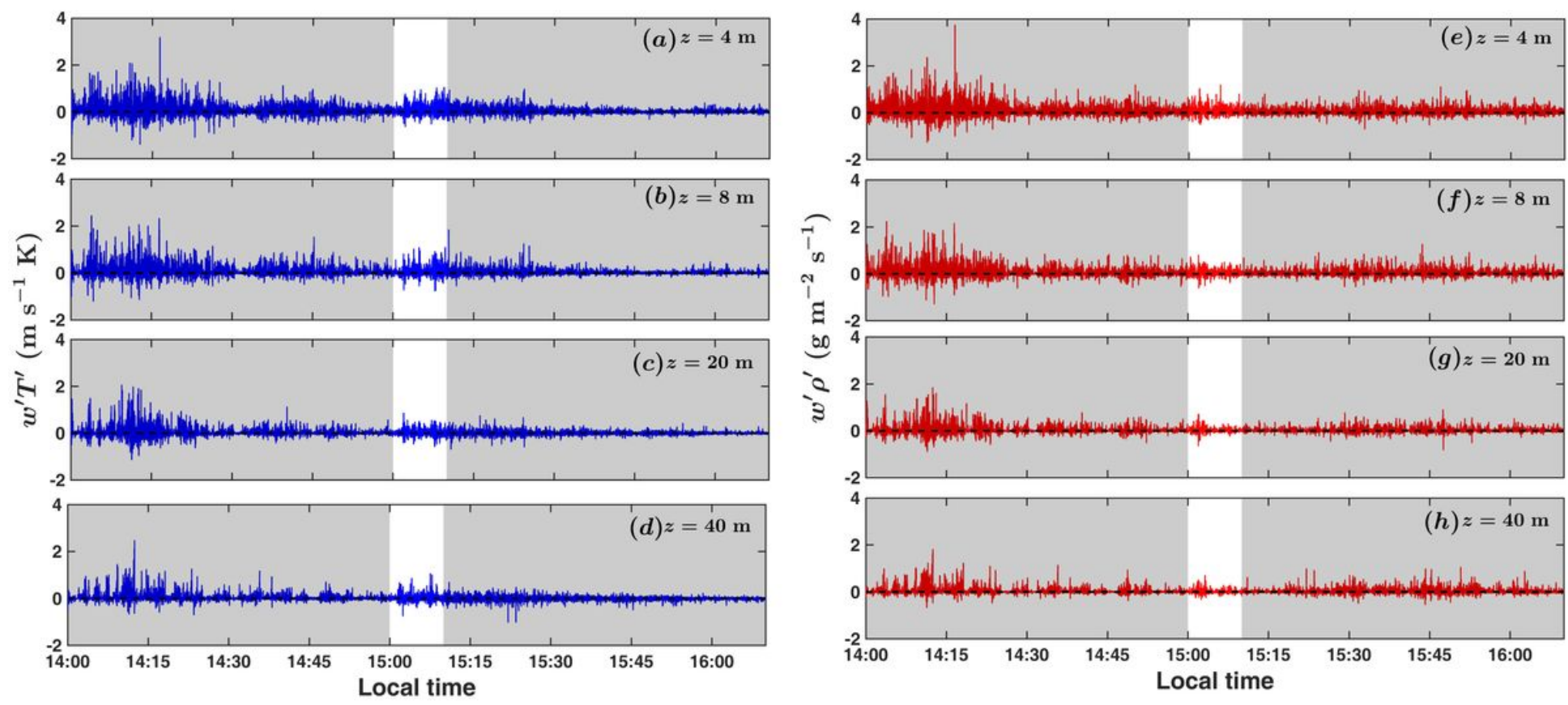

Figure 3

(see Manuscript file for full figure caption)
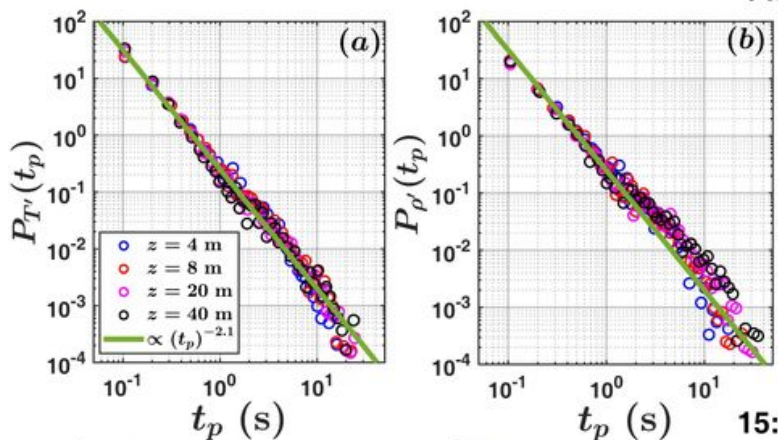

14:00-15:00
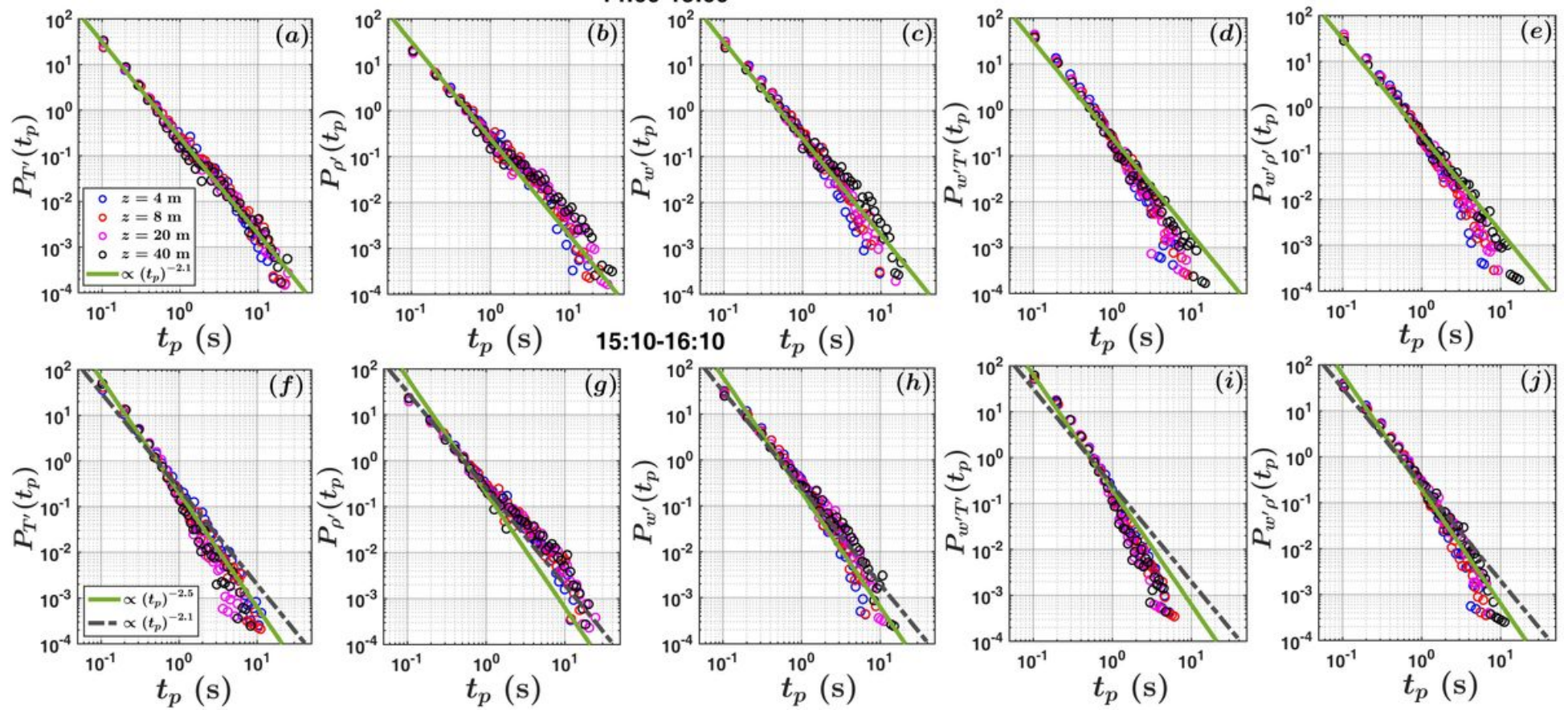

\section{Figure 4}

(see Manuscript file for full figure caption) 
14:00-15:00
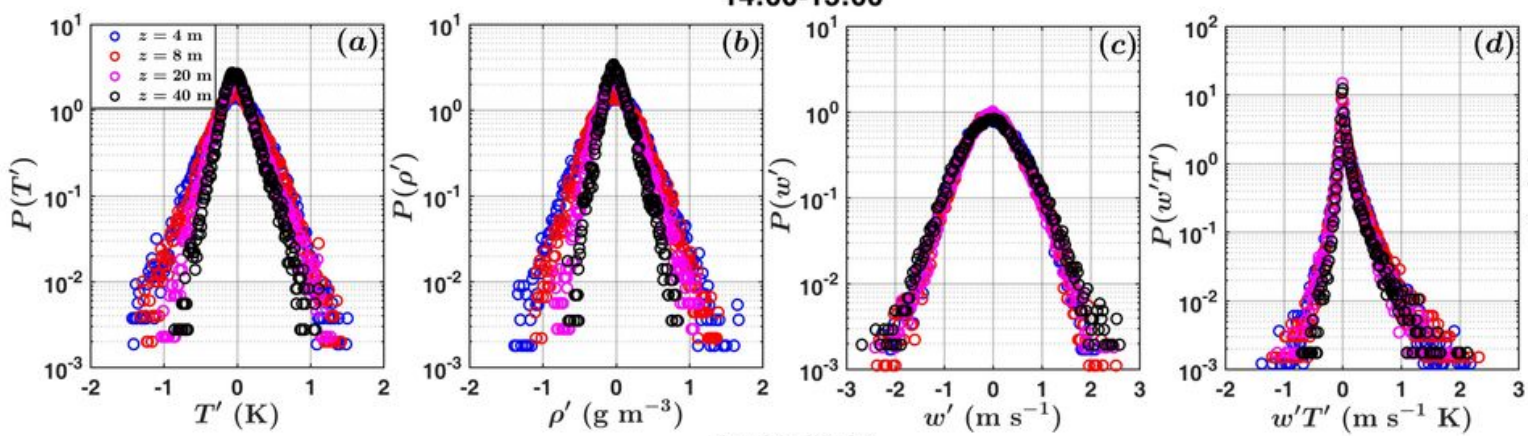

15:10-16:10
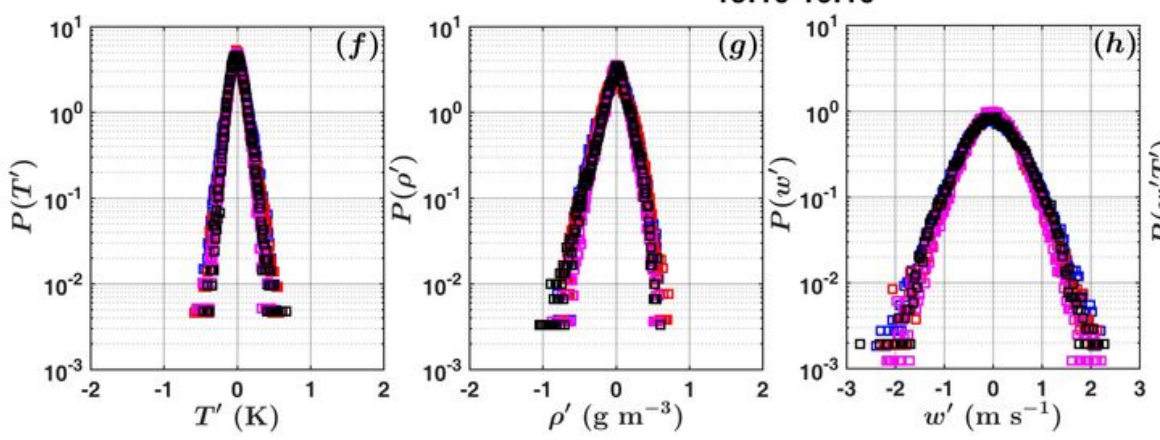
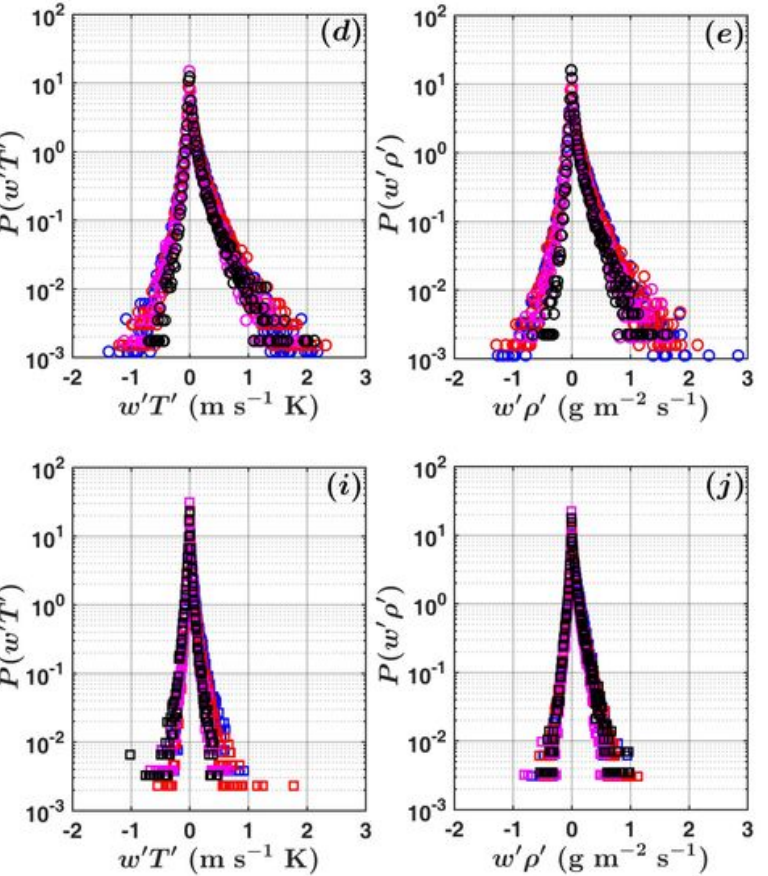

Figure 5

(see Manuscript file for full figure caption)
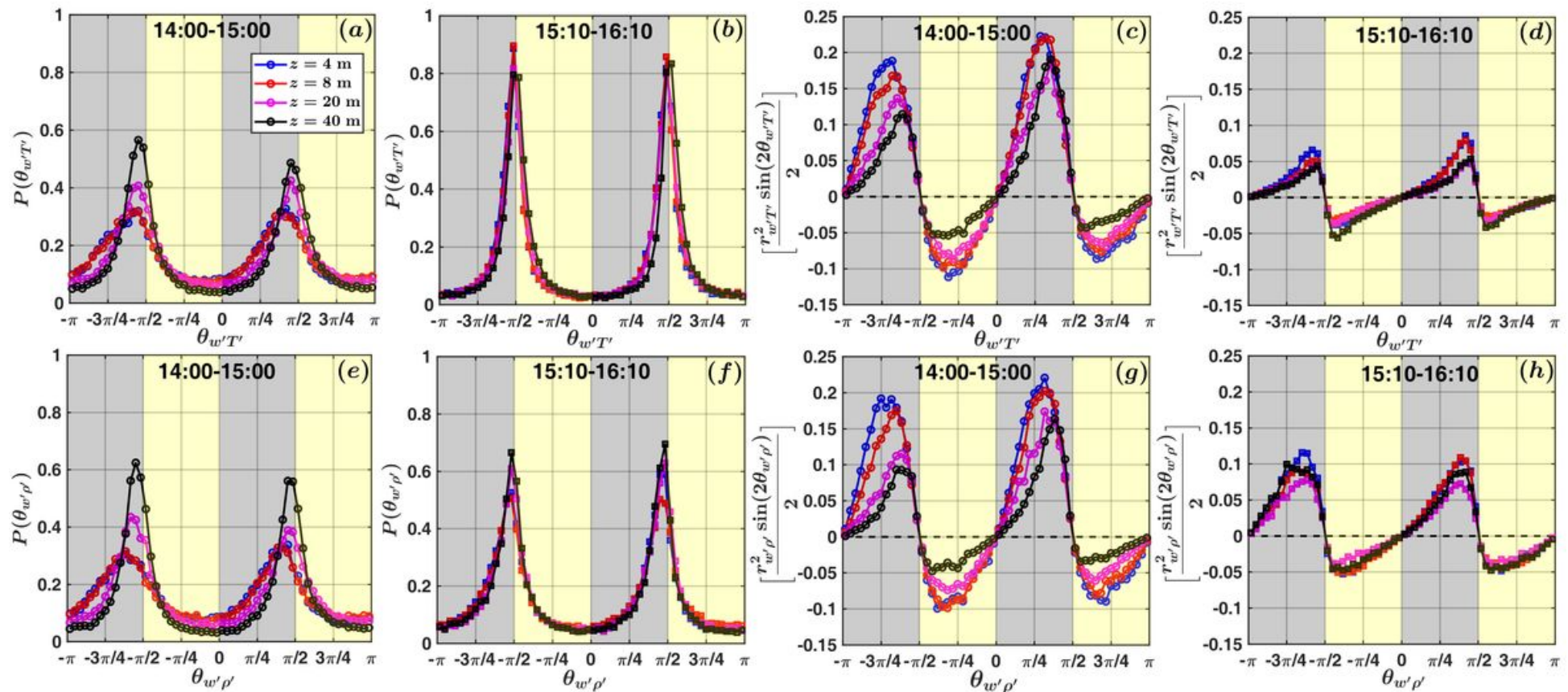

Figure 6

(see Manuscript file for full figure caption) 

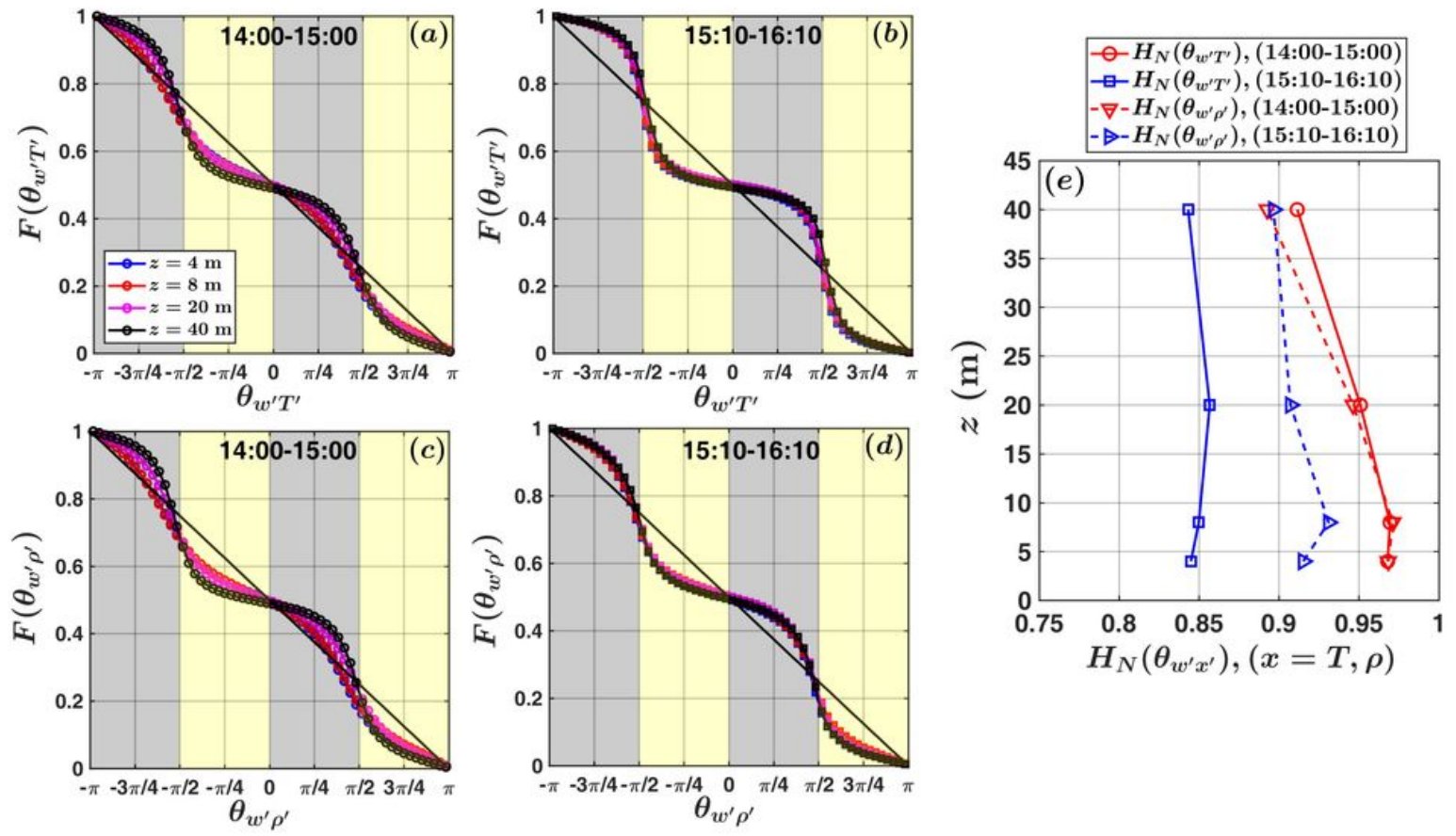

Figure 7

(see Manuscript file for full figure caption) 